\title{
Recent advances in the application of the heck reaction in the synthesis of heterocyclic compounds: An update
}

\author{
Majid M. Heravi ${ }^{1}$, Razieh, Moradi, Masoumeh, Malmir \\ ${ }^{1}$ Department of Chemistry, Faculty of Physics \&Chemistry Alzahra University, Vanak, Tehran, Iran.
}

\begin{abstract}
Background: The reaction of aryl halides or vinyl halides with activated alkenes in the presence of Palladium-catalyst along with a base for the formation of the $\mathrm{C}-\mathrm{C}$ bond is called the Heck reaction. This reaction has been employed extensively in the synthesis of heterocyclic compounds. Methodology: We have highlighted these applications in 2010. Conclusion: This review aims to cover the recent application of the Heck reaction in the synthesis of heterocyclic compounds during a period from 2010 to 2017. Dedicated to my dearest “ TEEDA" on the occasion of her third birthday.
\end{abstract}

Keywords: Carbon-carbon bond formation, Catalysis, Heck reaction, Heterocyclic compounds, Palladium, Pd 